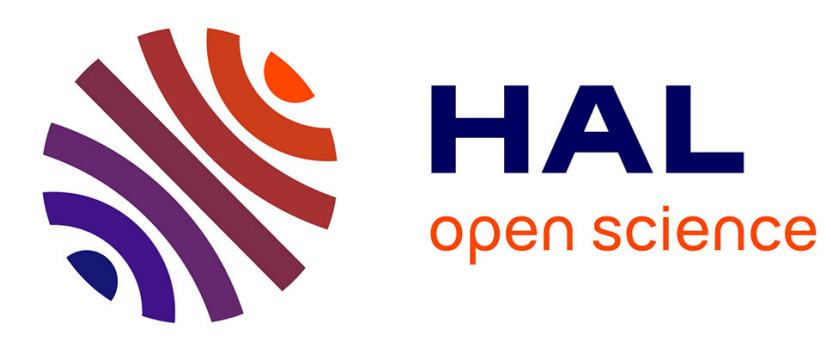

\title{
Object shape recognition using electric sense and ellipsoid's polarization tensor
}

Sylvain Lanneau, Vincent Lebastard, Frédéric Boyer

\section{To cite this version:}

Sylvain Lanneau, Vincent Lebastard, Frédéric Boyer. Object shape recognition using electric sense and ellipsoid's polarization tensor. 2016 IEEE International Conference on Robotics and Automation (ICRA), 2016, Stockholm, Sweden. pp.4692 - 4699, 10.1109/ICRA.2016.7487670 . hal-01503250

\section{HAL Id: hal-01503250 \\ https://hal.science/hal-01503250}

Submitted on 6 Apr 2017

HAL is a multi-disciplinary open access archive for the deposit and dissemination of scientific research documents, whether they are published or not. The documents may come from teaching and research institutions in France or abroad, or from public or private research centers.
L'archive ouverte pluridisciplinaire HAL, est destinée au dépôt et à la diffusion de documents scientifiques de niveau recherche, publiés ou non, émanant des établissements d'enseignement et de recherche français ou étrangers, des laboratoires publics ou privés. 


\title{
Object shape recognition using electric sense and ellipsoid's polarization tensor
}

\author{
Sylvain Lanneau ${ }^{1}$ and Vincent Lebastard ${ }^{2}$ and Frédéric Boyer ${ }^{3}$
}

\begin{abstract}
This paper deals with the geometrical properties of an ellipsoidal object (aspect ratio, volume, orientation) estimation with an underwater sensor inspired by the uncommon sense of the electric fish. The proposed method first locates the object independently of its geometrical properties thanks to the MUSIC (MUltiple SIgnal Classification) algorithm and then, estimates the geometrical properties using an optimization method and the object's electrical response model. The simulation results show the relevance of the method.
\end{abstract}

\section{INTRODUCTION}

In the field of underwater robotics, a big challenge consists in giving autonomy to robots navigating in turbid water and confined or post catastrophic environment. In such conditions, usual solutions such as vision and sonar are not efficient enough for trajectory control or cartography. But an alternative is developing since the early 2000's, by taking inspiration of the electric sense, a way of environment perception used by some fish which live in muddy waters and hunt by night (Gnathonemus petersii in Africa or Eigenmannia virescens in South America). This original sense has been discovered by biologists in the 50's [1]. The fish polarizes its body thanks to a dedicated organ and a surrounding electric field is thus created. The electrical field response of the objects in the neighborhood to that field is caught by the fish with a high density distribution of electroreceptors, spread out over its skin. The fish decodes this electric image [2] to recover the environment features. By observation and comprehension of these biological mechanisms, researchers in bio-inspired robotics designed new kinds of sensors directly inspired by electric sense. Simply composed of two emitting and two receiving electrodes at the beginning [3], researchers made these systems evolve toward more complex sensors. Nowadays, the sensors are cylindrical multi-electrodes devices using either $U-I$ technology (voltage polarization and current measurements) [4], [5], [6] or $U-U$ technology (voltage polarization and voltage measurements) [7]. The $U-I$ sensor, which is the purpose of this paper, is able to accurately estimate a sphere's location and size thanks to a Kalman filter algorithm [8]. With this technique, location and size are estimated at the same time. Indeed, we know the object's shape and position informations, both contained in the measured currents, to be intimately related to each other in a non-linear relationship. It is clearly shown by the Rasnow's potential perturbation model for a sphere of radius

$1,2,3$ Sylvain Lanneau, Vincent Lebastard and Frédéric Boyer are with IRCCyN, Ecole des Mines des Nantes, 4 Rue Alfred Kastler, 44300 Nantes, France $\{$ sylvain.lanneau, vincent. lebastard, frederic.boyer\}amines-nantes. fr $a$ and of center $O$ [9]:

$$
\delta \phi(M)=\chi\left(\frac{a}{r}\right)^{3} \mathbf{E}_{0} \cdot \mathbf{r}
$$

where $\delta \phi(M)$ is the potential perturbation evaluated at $M$ point, $\chi$ is the contrast factor, representing the relative object conductivity with respect to that of the water, $\mathbf{E}_{0}$ is an external and uniform electric field in the whole sphere, $\mathbf{r}=\overrightarrow{O M}$ and $r=\|\mathbf{r}\|$. A recent work [10] showed the feasibility, in the case of an ellipsoidal object, of location and geometrical informations extraction using the $U-U$ technology SensorPod probe. The algorithm estimates the object's features in a sequential process, by an active alignment of the sensor with respect to the object's main axis and suitable models for parameters estimation. Ellipsoidal objects are in fact of particular interest. The ellipsoid is the next shape after the sphere (and including it) in term of complexity. Moreover, [11], [12] showed that the response of a compact object to a uniform electric field can be approximated, at first order, by that of an ellipsoid. We propose here a new method for estimating the geometrical parameters of a prolate ellipsoid (one long axis and two shorter axis), using our $U$-I technology probe. It consists in the estimation of three parameters. The two first ones are those which uniquely define the ellipsoid. They can either be the major semi-axis $a$ and the minor semi-axis $b$, or the aspect ratio $\eta=a / b$ and the volume $V$. The third parameter is the pose angle between the sensor main axis and the ellipsoid semi-major axis, denoted $\theta_{0}$ (see figures $1 \mathrm{~b}$ and $1 \mathrm{c}$ ). The approach is based on methods recently introduced in the field of applied mathematics [13], in which electrolocation and shape recognition is numerically addressed with a dense array of electroreceptors. It consists in separating position and geometry informations from the sensor's input currents. This is performed in two steps : 1) locate the object independently of its intrinsic properties using the MUSIC (MUltiple SIgnal Classification) algorithm [14], [15] ; 2) knowing the position, estimate its geometrical properties with a least square optimization algorithm and a suitable equations system solving method. See a scheme of the process on figure 3 . The results introduced in this paper, obtained in simulation, demonstrate that the method can be implemented with our sensor, even though it has much less electrodes compare to [13]. These results are particularly encouraging and constitute a first step before their experimental implementation. After having described in details the sensor and the test bed in section II, the currents models in section III and the assumptions about the sensor and the objects in section IV, results on localization are 


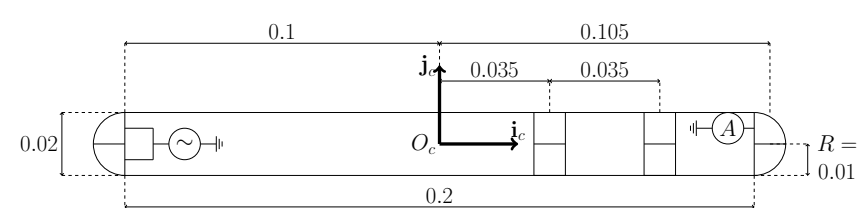

(a) Top view of the sensor

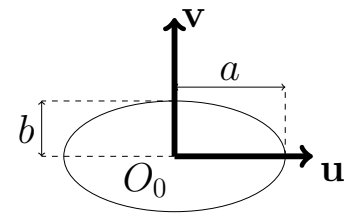

(b) Top view of the object

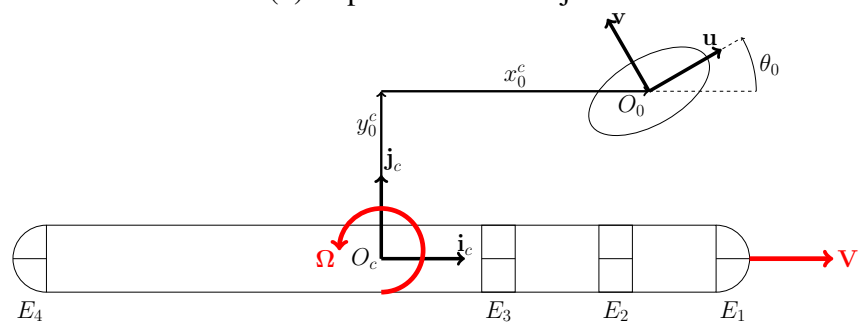

(c) Top view of the scene

Fig. 1: (a) The sensor with the polarization and measurement schematic and dimensions in meters. $R$ denotes the sensor radius. (b) The ellipsoid and its related local frame $\left(O_{o}, \mathbf{u}, \mathbf{v}\right) . O_{o}$ coincides with the geometrical center of the object. Vectors $\mathbf{u}$ and $\mathbf{v}$ are aligned with the semi-major axis $(a)$, and the semi-minor axis (b), respectively. (c) The sensor, the object and their respective frames. The coordinates of the geometrical center of the object are denoted $\left(x_{0}^{c}, y_{0}^{c}\right)$ in the sensor frame. Its orientation relative to the sensor axis is $\theta_{0}$. Vector $\mathbf{V}$ is the longitudinal velocity and vector $\boldsymbol{\Omega}$ is the rotation velocity about the vertical axis. The macroelectrodes are numbered from $E_{1}$ to $E_{4}$

presented in section $\mathrm{V}$ and on shape recognition in section VI. Section VII deals with future works for improvements and experimental implementation of the approach.

\section{THE ELECTROLOCATION TEST BED}

\section{A. Sensor}

The sensor is called slender probe due to its large aspect ratio and is composed of four macro-electrodes spaced by cylindric insulating parts, see figure 1a. Each macroelectrode can be set under an electric potential with respect to the others and this potential difference leads to an electric current flowing through all the conducting medium. In particular, the electric current flowing through the individual electrodes that compose each of the macro-electrodes can independently be measured with a remote Dspace ${ }^{\mathrm{R}}$ acquisition device (12 bits resolution, $5 \mathrm{~Hz}$ sampling frequency).

\section{B. Tank and Cartesian robot}

The probe described above is immersed into a $1 \mathrm{~m}$ sided cube tank. On the top of this tank, a Cartesian robot allows controlled translations along horizontal $x$ and $y$ axes and

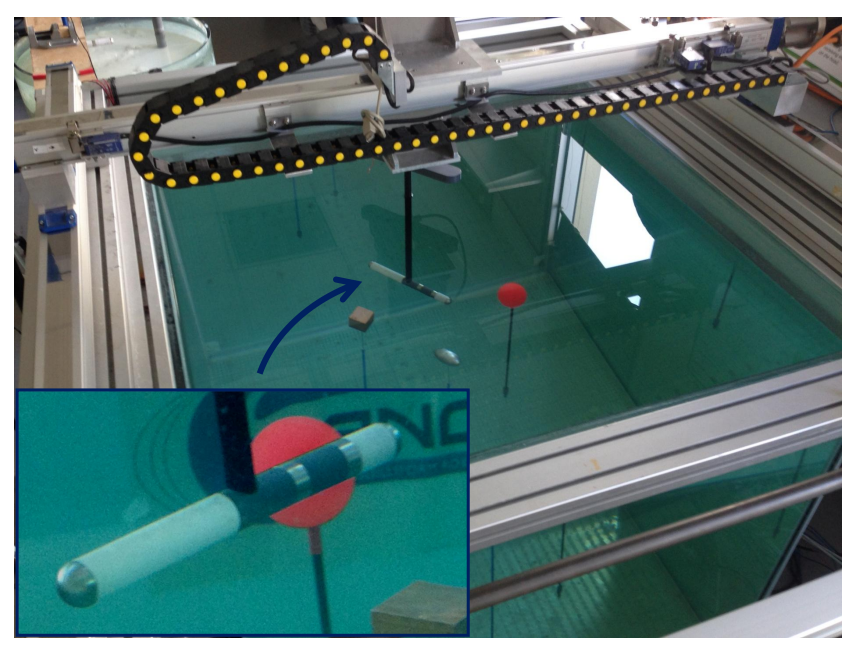

Fig. 2: The water tank topped with the Cartesian robot, permitting a planar motion of the sensor. A scene is composed of the sensor and objects lying in the same plane.

rotation about the vertical $z$ axis, see figure 2 . The motion control is performed with a DSpace ${ }^{\circledR}$ system with custom program. The sensor being linked to this robot thanks to a rigid epoxy fiber tube, its motion in a plan can be controlled. For a complete description of the robot (electronics schematics, noise level, positioning precision, sensitivity to temperature) refer to [5]. A scene can be composed by placing some objects of different shapes, sizes and materials at different locations in the tank.

\section{CURRENTS MODELS}

The model of currents describes the direct problem, which consists in knowing the electric currents flowing through the electrodes for a given scene, defined by the sensor's, the objects' and the medium's characteristics. In fact, we have at our disposal two models for the direct problem. They are both entirely described in [4] and we briefly present them in this section. The first one, called BEM (standing for Boundaries Elements Method), is derived from the electrostatics theory and the currents are computed thanks to a discretization in finite elements of all the boundaries of the scene. It provides a numerical solution of the direct problem [16], which will be used as the sensor input currents in this paper (input data of the process, as shown on figure 3 ). The second one, known as the analytical model, describes the measured currents with simple explicit mathematical expressions deduced from a reduction process described in [4]. The methods used for the localization and shape recognition are based upon this analytical model, see figure 3.

\section{A. BEM Formulation of the direct problem}

An electric potential difference is imposed between the electrodes of the sensor, so there exists an electric potential $\phi$ in the surroundings, satisfying Laplace equation : $\Delta \phi=$ 0 . All over the scene, Ohm's law is locally described by 


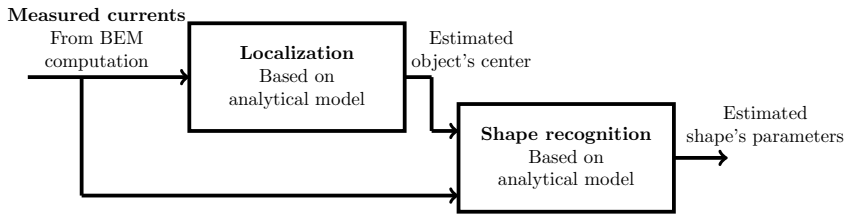

Fig. 3: Scheme of the process. The BEM computed currents are used as input. The methods for localization and shape recognition are based upon the analytical model.

$\mathbf{j}=\gamma \nabla \phi$, j being the current density $\left(A / m^{2}\right)$ and $\gamma$ the medium conductivity $(S / m)$. Boundary conditions on the sensor and the objects are known, depending on their material dielectric properties (conducting or insulating). The current flowing through the boundaries of each electrode is defined as the flux of the normal (with respect to the surface) current density. Thanks to the discretization of all the boundaries of the scene, the currents flowing through each electrode is numerically computed. BEM computed currents faithfully estimate the measured currents with the true sensor's geometry [4]. BEM computed currents will serve as input currents in this paper, in preparation to their substitution by future experiments.

\section{B. Analytical model}

Let us now add some assumptions: 1) the sensor has the slender shape and the electrodes are arranged as described in section II-A ; 2) each object is small enough to assume the electric field to be uniform over its whole volume. In practice, constraint 2) is justified when the lateral distance between sensor main axis and object's center is equal or greater than three sensor radius $\left(y_{0}^{c} \geq 3 R\right.$ on figure 1) and when the size of the object is at most of the order of the sensor radius $(a \leq R)$ [4]. Under these assumptions, the following analytical model for the currents is established. It describes a linear relationship between the vector $U \in$ $\mathbb{R}^{4}$, being defined as the electric potentials we can set onto macro-electrodes, and two currents vectors denoted $I_{\text {ax }} \in \mathbb{R}^{4}$ and $I_{\text {lat }} \in \mathbb{R}^{4}$

$$
\begin{aligned}
& I_{\text {ax }}=\left(C^{0}+\delta C\right) U \\
& I_{\text {lat }}=\frac{1}{4 \pi} P_{\perp} H P^{c} G^{\mathrm{T}} C^{0} U .
\end{aligned}
$$

Each component of the vector $I_{\mathrm{ax}}$ (resp. $I_{\text {lat }}$ ) is the "axial current" (resp. "lateral current") of the corresponding macroelectrode [4]. In particular, the model of the $I_{\mathrm{ax}}$ vector (2) shows a decomposition into two independent contributions. First, $I^{0}=C^{0} U$ is the axial currents vector measured in the case where there is no object in the scene. The $4 \times 4$ real matrix $C^{0}$ represents the conductivity of the surroundings which with no object only depends on the sensor's geometry and the medium's conductivity $\gamma$. It is obtained once for all either by numerical computation or by a calibration phase. Second, $\delta I_{\mathrm{ax}}=\delta C U$ is the axial perturbation vector induced by the presence of the object and the $4 \times 4$ real matrix $\delta C$ is the conductance variation matrix that encodes the surroundings' conductance variation due to the presence of the object. A model for $\delta C$ is given in [4] and here rewritten as

$$
\delta C=\frac{1}{4 \pi \gamma} C^{0} G P^{c} G^{\mathrm{T}} C^{0} .
$$

The $4 \times 3$ real matrix $G$ is a non-linear function of the object's coordinates in the sensor frame, whereas the matrix $P^{c}$ depends on object's properties only (detailed in section IV). In the signal array processing literature [15], $\delta C$ is known as the multistatic response matrix. On the other hand, the analytical expression (3) of the $I_{\text {lat }}$ vector handles a 4 $\times 4$ real matrix $P_{\perp}$ which depends on the sensor's only. It describes how the macro-electrodes are laterally polarized under the effect of the electric field reflected by the object. The $4 \times 3$ real matrix $H$, which, as the matrix $G$, is a non-linear function of the object's coordinates in the sensor frame. In practice, the components of $I_{\mathrm{ax}}$ (resp $I_{\text {lat }}$ ) are obtained by computing the sum (resp. the difference) of the currents flowing across the two corresponding left and right electrodes. Thus, a numerical value of the matrix $\delta C$ for a given scene can be obtained. Remarking that the measured vector $\delta I_{\mathrm{ax}}$ for a polarization vector $U=\left(\begin{array}{llll}1 & 0 & 0 & 0\end{array}\right)^{\mathrm{T}}$ equals to the first column of $\delta C$, the four columns can be recovered by successively setting each macro-electrode to $1 V$ and the three others to $0 \mathrm{~V}$.

\section{HYPOTHESES AND REQUIREMENTS}

\section{A. Additional assumptions}

In addition to the basic assumption of the analytical model (previous section), let us add further restrictions on the sensor motion and on the objects' features : 1) the sensor always moves in straight line, along its main axis : rotation velocity $\Omega$ shown on figure $1 \mathrm{c}$ is set to zero ; 2 ) there is only one object at each time in the scene and it lies in the same plan as the sensor ; 3) the object's material properties (perfectly conducting or perfectly insulating) and the side of the sensor on which the object is, are determined by an algorithm that use the signs of the currents $\delta I_{\mathrm{ax}}$ and $I_{\mathrm{lat}}$, described in [17] ;4) the object is assumed to be ellipsoidal (or cubic, in order to assess the robustness of the method for other shapes) ; 5) the ellipsoids used in this paper are supposed to be prolate, that is, their rotation axis corresponds to their semi-major axis ; 6) the aspect ratio is $\eta=a / b, a$ being the semi-major axis, and $b$, the semi-minor axis, ranging from 1 (sphere) to $2.5 ; 7)$ all the objects' surfaces are smooth, with no cracks. The objects used in this paper are described in table I. The problem being symmetric with respect to the sensor's main axis, the objects are placed on the left side of the sensor only.

\section{B. A model for the object's electrical response}

According to [11], [12], the response of an ellipsoidal object to a uniform electric field is characterized by the first order polarization tensor which, in the object's principal basis, can be written as a $3 \times 3$ diagonal matrix, denoted $P$. An analytical expression of $P$ is given in [12]. For objects with large conductivity (conducting) or small conductivity 


\begin{tabular}{|c|c|c|c|c|c|c|}
\hline & $\boldsymbol{x}$ & $\boldsymbol{x}$ & $\otimes$ & $\otimes$ & $\otimes$ & $\times$ \\
\hline Number & 1 & 2 & 3 & 4 & 5 & 6 \\
\hline Shape & Cube & Cube & Ellipsoid & Ellipsoid & Ellipsoid & Sphere \\
\hline $\begin{array}{c}x_{0} \\
(\mathrm{~cm})\end{array}$ & 0 & 11 & 7 & -4 & 13 & \pm 20 \\
\hline $\begin{array}{c}y_{0} \\
(\mathrm{~cm})\end{array}$ & 6 & 8 & 7 & 9 & 5 & 3 to 10 \\
\hline$a(\mathrm{~cm})$ & 1 & 1 & 0.8 & 1 & 1 & 1 \\
\hline$b(\mathrm{~cm})$ & 1 & 1 & 0.67 & 0.5 & 0.4 & 1 \\
\hline$\eta$ & 1 & 1 & 1.2 & 2 & 2.5 & 1 \\
\hline $\begin{array}{c}V \\
\left.(\mathrm{~cm})^{3}\right)\end{array}$ & 8 & 8 & 1.5 & 1.05 & 0.67 & 4.2 \\
\hline Material & Insul. & Cond. & Cond. & Insul. & Cond. & Cond. \\
\hline $\begin{array}{c}\theta_{0} \\
(\mathrm{deg})\end{array}$ & 30 & 0 & -20 & -70 & 35 & - \\
\hline
\end{tabular}

TABLE I: The objects' representations and their corresponding features. $\left(x_{0}, y_{0}\right)$ are the coordinates of the object in the fixed frame. For the cubes, semi-axis $a$ and $b$ are defined as half of the edge length.

(insulating) compare to that of the water, $P$ only depends on the object's volume and aspect ratio through the expression

$$
\begin{aligned}
& P=V\left(\begin{array}{ccc}
f(\eta) & 0 & 0 \\
0 & g(\eta) & 0 \\
0 & 0 & g(\eta)
\end{array}\right) \\
& \begin{cases}f=\frac{1}{A}, g=\frac{1}{B} & \text { for a conducting object } \\
f=\frac{1}{A-1}, g=\frac{1}{B-1} & \text { for an insulating object }\end{cases}
\end{aligned}
$$

with functions $A$ and $B$ being defined as

$$
\begin{aligned}
A, B: \mathbb{R} & \rightarrow \mathbb{R} \\
A(\eta) & =\eta^{-2} \int_{1}^{+\infty} \frac{1}{t^{2}\left(t^{2}-1+\eta^{-2}\right)} d t, \\
B(\eta) & =\eta^{-2} \int_{1}^{+\infty} \frac{1}{\left(t^{2}-1+\eta^{-2}\right)^{2}} d t .
\end{aligned}
$$

Relatively to the sensor main axis, the ellipsoid is rotated of an angle $\theta_{0}$ about the vertical axis. So, in the sensor frame, the matrix $P$ becomes

$$
P^{c}=R_{\theta_{0}} P R_{\theta_{0}}^{\mathrm{T}}
$$

the subscript $\mathrm{T}$ denoting the transposition and $R_{\theta_{0}}$ being the rotation matrix of angle $\theta_{0}$ about the vertical axis. The matrix product (6) leads to the following expression for the components of $P^{c}$

$$
\begin{aligned}
& p_{11}^{c}=p_{11} \cos ^{2}\left(\theta_{0}\right)+p_{22} \sin ^{2}\left(\theta_{0}\right), \\
& p_{12}^{c}=\left(p_{11}-p_{22}\right) \cos \left(\theta_{0}\right) \sin \left(\theta_{0}\right), \\
& p_{22}^{c}=p_{11} \sin ^{2}\left(\theta_{0}\right)+p_{22} \cos ^{2}\left(\theta_{0}\right) .
\end{aligned}
$$

The component $p_{i j}^{c}$ (reps. $p_{i j}$ ) denotes the $i^{\text {th }}$ row and $j^{\text {th }}$ column component of $P^{c}$ (resp. $P$ ). With the symmetry properties of the problem, we have $p_{21}^{c}=p_{12}^{c}$, while $p_{33}^{c}=p_{33}$ and plays no role in the problem. The others components equal to zero. For a straight line motion of the sensor sweeping past the object 5 (table I), and with a constant polarization vector $U=\left(\begin{array}{llll}0 & 0 & 0 & 1\end{array}\right)^{\mathrm{T}}$, the $\mathrm{BEM}$
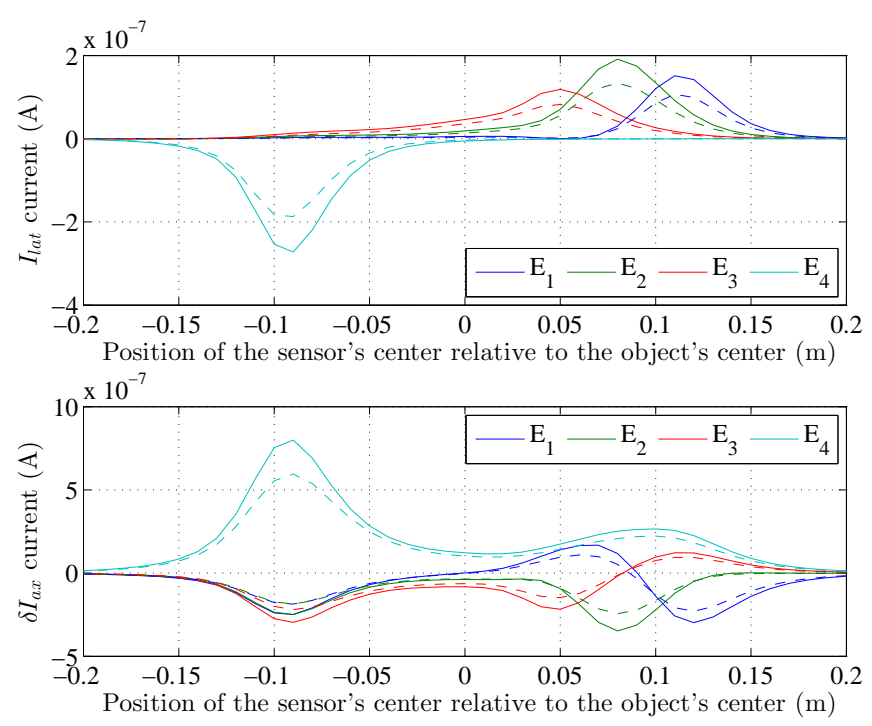

Fig. 4: Currents $I_{\text {lat }}$ (top) and $\delta I_{\text {ax }}$ (bottom) for a straight line motion of the sensor, with constant polarization vector $U=\left(\begin{array}{llll}0 & 0 & 0 & 1\end{array}\right)^{\mathrm{T}}$, going past the object numbered 5 in table $\mathrm{I}$. Solid lines : BEM currents. Dashed lines : currents calculated with the analytical model.

currents (solid lines) and the currents computed with the analytical model (dashed lines) are superimposed on figure 4. Even though there are amplitudes differences, the analytical model faithfully describes the BEM computed currents. Now the context of the study being fixed, the description of the shape recognition process can start.

\section{LOCALIZATION}

As explained in introduction, the first step before shape recognition is the localization of the object independently of its intrinsic properties. This is in agreement with biologists' studies who showed the ability of the fish to localize objects independently of their shapes [18]. Moreover, recent results on electric fish numerical modeling showed the feasibility of object's localization separately from of its intrinsic properties by using the MUSIC algorithm [13], [19]. This section details the implementation of the MUSIC algorithm to our sensor.

\section{A. Model-based localization : the MUSIC algorithm}

MUSIC is a model-based sensor array signal processing algorithm [20]. It has been developed since the 80's to estimate the direction of arrivals of electromagnetic and sound waves onto a passive antenna network [14]. In the 2000's, [15] showed that the principle could be extended to an active antenna network in order to localize scatterers in an homogeneous medium. By sequentially emitting a polarization wave with each antenna of the network and by measuring the polarized scatterers' responses with the whole network, the multistatic response (MSR) matrix is computed. In our case, the MSR matrix is the conductance variation matrix $\delta C$ (4). Then, a Singular Value Decomposition (SVD) 
Trajectories of the steering vectors.

They are functions of the candidate coordinates and determined by the analytical model

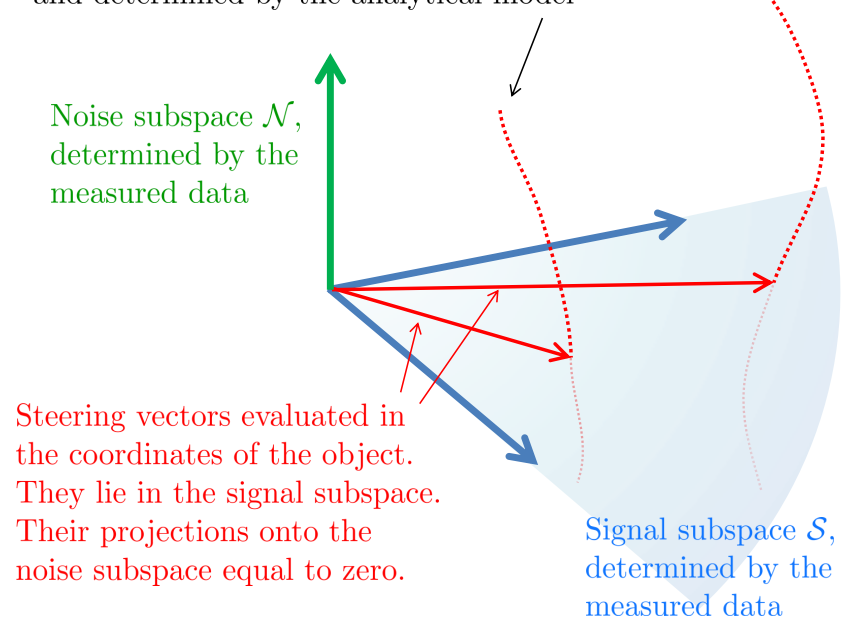

Fig. 5: Geometric representation of the MUSIC principle in dimension 3 (inspired by fig.1 in [14]). A basis of the noise subspace $\mathcal{N}$ is obtained with the SVD of the measured matrix $\delta C$. A set of steering vectors, corresponding to a set of candidate space coordinates are computed, defining trajectories represented in dotted lines. In virtue of the orthogonality between $\mathcal{S}$ and $\mathcal{N}$, the steering vectors which projection onto $\mathcal{N}$ is 0 belong to $\mathcal{S}$. The corresponding space coordinates are taken as an estimation of the object's center location.

of this matrix is performed [21]. The singular vectors associated to non-zero singular values form a basis of the signal subspace $\mathcal{S}$ whereas the singular vectors associated to zero eigen values form a basis of its orthogonal complement, the noise subspace $\mathcal{N}$. Figure 5 represents these two subspaces in dimension 3 (the dimension is in fact 4 in our case because we have 4 components currents vectors). On the other hand, from the analytical model of the MSR matrix, one can exhibit particular vectors, called steering vectors in the literature [15]. In fact, they correspond to the Green function vectors of the background medium. Steering vectors have two important properties: first, they are functions of the three space coordinates and they are independent of scatterers' intrinsic properties ; second, when evaluated in the scatterers' coordinates they are known to lie in the subspace $\mathcal{S}$. So, in virtue of the orthogonality between the subspaces $\mathcal{S}$ and $\mathcal{N}$, the projection onto $\mathcal{N}$ of the steering vectors evaluated in the object's coordinates tends toward 0 . Figure 5 describes how MUSIC makes good use of this fact in practice. Moreover, an implementation of the MUSIC algorithm is precisely described in [22] for the localization of buried unexploded ordnance. Even though the information carrier is a magnetic (not electric) field and the sensor array is planar (not linear), the analytical model for our respective MSR matrices are of identical structures. So, by using a similar process, we implemented the MUSIC algorithm with

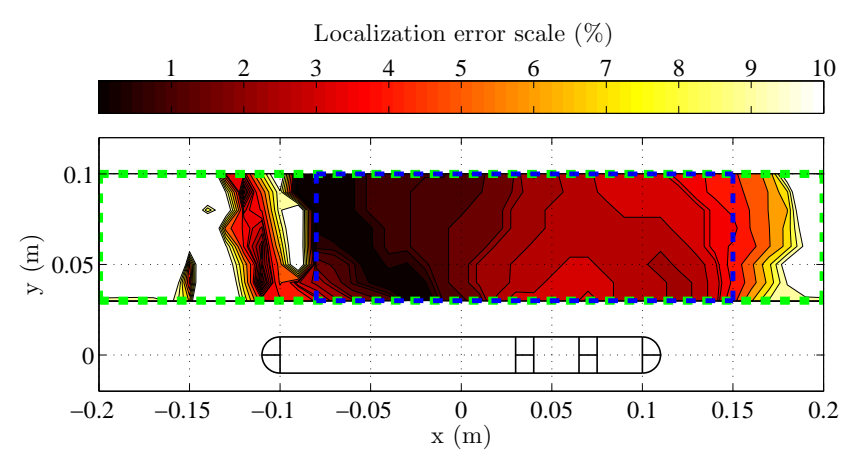

Fig. 6: MUSIC localization error map in percentage of sensor length $(22 \mathrm{~cm})$ for a $1 \mathrm{~cm}$ radius conducting sphere placed inside the green dashed line delimited area. Blue dotted line roughly defines an area in which the error is less than $5 \%$.

our sensor model according to the main following steps:

- Measure the MSR matrix $\delta C$ by switching the polarized macro-electrode one after the other, as described in section III-B.

- Compute the SVD of the matrix $\delta C$.

- Select the singular vectors associated with the lowest singular values in order to form a basis of the noise subspace $\mathcal{N}$.

- A set of candidate coordinates are defined by discretization of an area around the sensor, in the plan in which the sensor and the object lie.

- For each node of this grid, evaluate the steering vectors in the coordinates of that node and compute the inverse of their orthogonal projection onto $\mathcal{N}$.

- Once all nodes have been treated, select the node for which the inverse of the projection is the highest and take as the object location the coordinates of that node.

\section{B. MUSIC algorithm localization performances}

To evaluate the performance of the MUSIC algorithm for localization, an error map was computed for a $0.01 \mathrm{~m}$ radius conducting test sphere (object 6 in table I), see figure 6 . This map is obtained in the following manner. First, the sensor is placed so that the sensor's frame and the fixed frame coincide. An area of size $0.4 \mathrm{~m} \times 0.07 \mathrm{~m}$ is defined on the left side of the sensor (see green dashed rectangle on figure 6). Then, this area is discretized by a $0.01 \mathrm{~m}$ square grid and the sphere is placed at each node of this grid. For each sphere position on the grid, the MUSIC algorithm is applied with a $1 \mathrm{~mm}$ square discretized search area. Finally, for each sphere position a relative location error $\epsilon$, in percentage of the sensor's over all length is computed according to

$$
\epsilon=100 \cdot \sqrt{\left(\hat{x}_{0}^{c}-x_{0}^{c}\right)^{2}+\left(\hat{y}_{0}^{c}-y_{0}^{c}\right)^{2}} / 0.22,
$$

$\left(x_{0}^{c}, y_{0}^{c}\right)$ and $\left(\hat{x}_{0}^{c}, \hat{y}_{0}^{c}\right)$ being the true and the estimated coordinates of the center of the object in the sensor's frame, respectively. One can observe that the best results are obtained in a longitudinal area ranging from $-0.02 \mathrm{~m}$ to $-0.08 \mathrm{~m}$, in which the error is less than $2 \%$. More generally, the area 


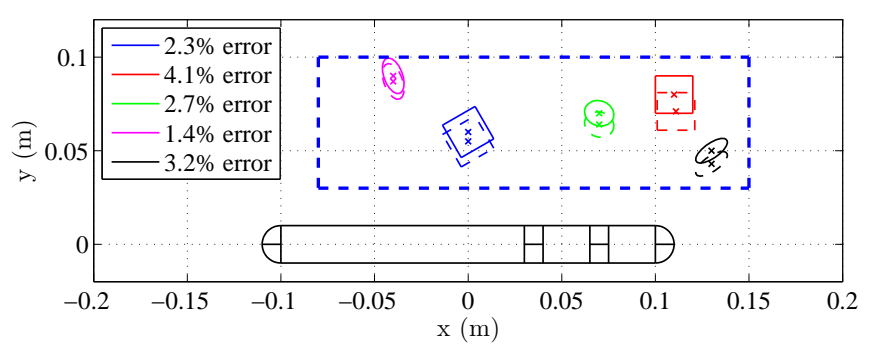

Fig. 7: Localization error in percentage of sensor diameter for several cubes and ellipsoids using MUSIC algorithm. Solid lines : true objects' locations, dotted lines : estimated objects' locations. Blue dotted line is the same as that of figure 6 . Note that even though they are represented on the same figure, the objects were localized one after the other, not at the same time.

delimited by the blue dashed line defines a zone in front of the sensor where the localization error is less than 5\%. Recall that a $5 \%$ error corresponds to an absolute error of $1.1 \mathrm{~cm}$. If the sphere is outside of this area, the error can reach $10 \%$ or more (white zones). Then we have tested the algorithm with the other objects described in table I. The results on figure 7 and in table II show that the location errors are of identical magnitude compare to the case of the sphere. This confirms the ability of the MUSIC algorithm to estimate the position of the center of an object independently of its shape with our sensor design. In other terms, the location and geometrical informations have been separated, as expected.

\section{Shape RECOGNITION}

Once the object is located with the method described above, the purpose of this section is the estimation of the objects properties. The strategy for shape recognition will consists of two steps : first, we estimate the components of the matrix $P^{c}$ with measured currents ; second, we estimate the parameters $\theta_{0}, \eta$ and $V$ using the model of the components of $P^{c}$, described in (7) to (9).

\section{A. Matrix $P^{c}$ estimation}

As mentioned in [23], [13], our typical structure for the analytical model of $\delta I_{\mathrm{ax}}$ and $I_{\text {lat }}$ (section III-B) allows rewriting the currents vectors as a system of equations linear with respect to the components of $P^{c}$. In the literature [24], this model structure is known as linear regression, and a solution for the components of $P^{c}$ can be estimated with an optimization algorithm such as the well known least squares. This method requires a collection of measurement points. For the sake of simplicity, the straight line trajectory have been chosen, because it doesn't require any other motion but constant forward speed. The trajectory is $0.4 \mathrm{~m}$ long sweeping past the object and at a constant lateral distance. The polarization vector is constant along this trajectory: $U=$ $\left(\begin{array}{llll}0 & 0 & 0 & 1\end{array}\right)^{\mathrm{T}}$ and the currents vector $I_{\text {lat }}$ is measured every centimeter. Let us denote $\hat{P}^{c}$ the estimate of $P^{c}$ obtained this way. A comparison between BEM currents (solid lines)
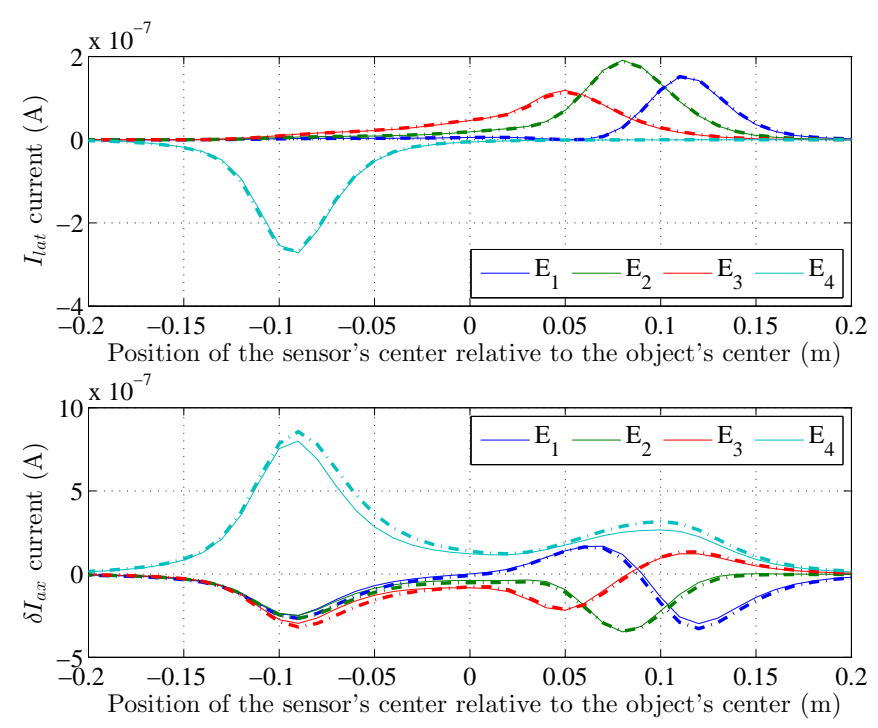

Fig. 8: Currents $I_{\text {lat }}$ (top) and $\delta I_{\text {ax }}$ (bottom) for a straight line motion of the sensor, with constant polarization vector $U=\left(\begin{array}{llll}0 & 0 & 0 & 1\end{array}\right)^{\mathrm{T}}$, going past the ellipsoid represented with black line in table I . Solid lines : BEM currents. Dash-dot lines : currents calculated with the analytical model in which $P^{c}=\hat{P}^{c}, P^{c}$ being estimated with the least squares method.

and modeled currents using the estimated matrix $\hat{P}^{c}$ (dashdot lines) is shown in figure 8 . The identification process provides a $\hat{P}^{c}$ matrix that makes the model to fit the input data (BEM) very well. In next section, we will see how the object's properties estimation can be performed, given an estimated matrix $\hat{P}^{c}$.

\section{B. Object's parameters estimation}

Having an estimation $\hat{P}^{c}$, the parameters $\theta_{0}, \eta$ and $V$ can be computed by solving the system of three nonlinear equations in three unknowns (7) to (9). For this, any numerical system as the function fsolve of the Matlab ${ }^{\circledR}$ software is appropriate. However, our intention being to implement this algorithm on autonomous system in the future, we propose another method requiring no particular computing software. The idea is to extract and estimate, by using the analytical model of $P^{c}$, each unknown one after the other. First, according to equations (7) to (9), and supposing the component $p_{12}^{c} \neq 0$ in a first step, the ratio $\frac{p_{11}^{c}-p_{22}^{c}}{p_{12}^{c}}$ is independent of $\eta$ and $V$. We have $\frac{p_{11}^{c}-p_{22}^{c}}{p_{12}^{c}}=h\left(\theta_{0}\right), h$ being defined as

$$
h:]-\frac{\pi}{2} ; 0[\cup] 0 ; \frac{\pi}{2}\left[\rightarrow \mathbb{R}, \theta_{0} \mapsto \frac{1-\tan ^{2}\left(\theta_{0}\right)}{\tan \left(\theta_{0}\right)} .\right.
$$

Function $h$ having two solutions over $]-\frac{\pi}{2} ; 0[\cup] 0 ; \frac{\pi}{2}[$ (see figure 9), the sign of the component $p_{12}^{c}$ is used to resolve this indetermination. In the particular case where the estimated component $\hat{p}_{12}^{c}$ is null, the matrix $\hat{P}^{c}$ is diagonal and corresponds to either a sphere or an ellipsoid with an angle of 0 or $\pi / 2$. As a result, the simple following algorithm ensures the discrimination between the three cases: 

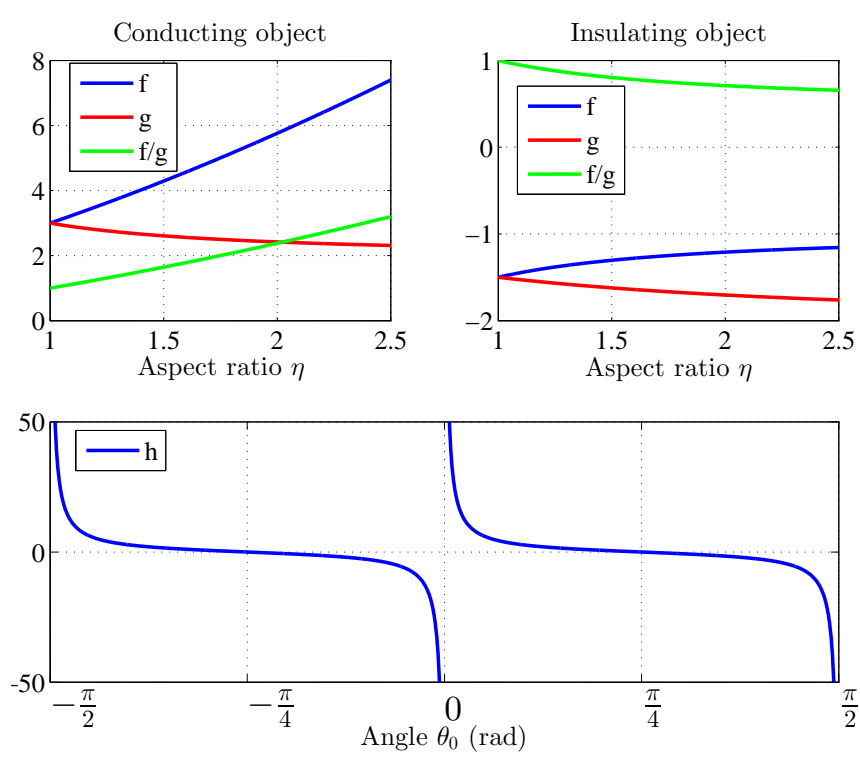

Fig. 9: Functions $f, g$ and $f / g$ for a conducting object (top left) and an insulating object (top right) over the interval $[1 ; 2.5]$. Function $h$ over the interval $\left[-\frac{\pi}{2} ; \frac{\pi}{2}\right]$ (bottom).

- If $\hat{p}_{11}^{c}=\hat{p}_{22}^{c} \Rightarrow$ object is a sphere $\Rightarrow \eta=1$.

- If $\hat{p}_{11}^{c}>\hat{p}_{22}^{c} \Rightarrow$ object is an ellipsoid with $\theta_{0}=0$.

- If $\hat{p}_{11}^{c}<\hat{p}_{22}^{c} \Rightarrow$ object is an ellipsoid with $\theta_{0}=\pi / 2$.

Then, a rotation matrix $\hat{R}_{\theta_{0}}$ is estimated and one can recover an estimation $\hat{P}$ of matrix $P$, by computing the matrix product

$$
\hat{P}=\hat{R}_{\theta_{0}}^{\mathrm{T}} \hat{P}^{c} \hat{R}_{\theta_{0}} .
$$

Next, from the tensor model (5), the ratio $\frac{p_{11}}{p_{22}}$ is a function of the aspect ratio $\eta$ only, and is independent of the volume $V$. Function $f / g$ having only one solution over the range $[1 ; 2.5]$ (see figure 9), the aspect ratio can be unambiguously estimated from this function (remind that the conductivity is already known, as mentioned in section IV, so there is no doubt about which curve has to be used). Finally, volume $V$ can be estimated using $\hat{p}_{11}$ or $\hat{p}_{22}$

$$
V=\frac{\hat{p}_{11}}{f(\hat{\eta})} \text { or } V=\frac{\hat{p}_{22}}{g(\hat{\eta})} .
$$

The shape recognition results are presented on figure 10 in the case where the localization error have not been introduced in the process. The cubes are approximated by ellipsoids with aspect ratios close to 1 , ie. spheres with a radius roughly equal to the half-diagonal of the cube face (ie. a radius of $\sqrt{2} \approx 1.41 \mathrm{~cm}$ ). This is in accordance with [11] in which a $2 \mathrm{~cm}$ sided cube is theoretically estimated by a $1.32 \mathrm{~cm}$ radius sphere. In spite of an over estimation of the sizes (except for the sphere), the ellipsoidal objects are faithfully estimated. This proves, at least in simulation, that the geometrical parameters estimation algorithm works if the location is well known. The over estimated volumes for the ellipsoids and cubes can be attributed to the purely dipolar object's response hypothesis of the analytical model. The
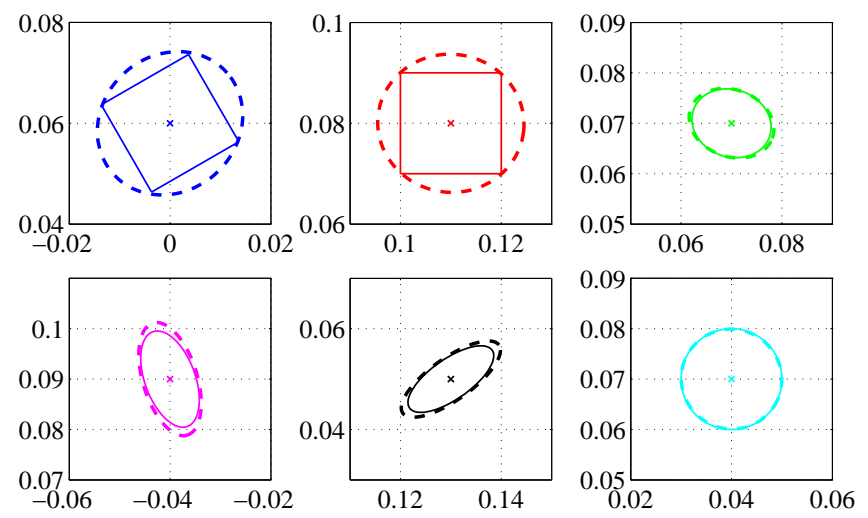

Fig. 10: Comparison between real objects (solid lines) and estimated objects (dashed lines) with no localization in the shape recognition process. Colors correspond to those of the table I. All dimensions in meter.
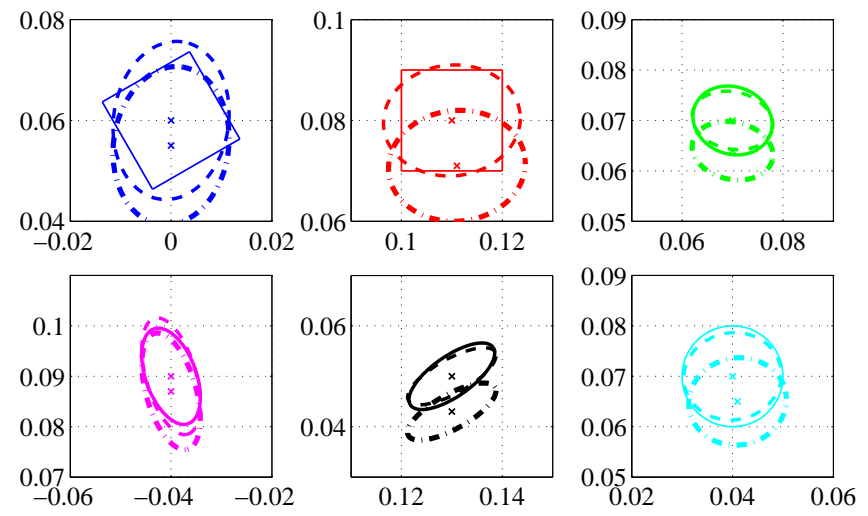

Fig. 11: Comparison between real objects (solid lines) and estimated objects (dashed lines and dash-dot lines) with MUSIC algorithm localization error taken into account in the shape recognition process. Dashed lines represent same ellipses as the dotted ones but placed at the true object's location for shape recognition error visualization only. Colors correspond to those of the table I. All dimensions in meter.

BEM formulation does not make such assumption, resulting into BEM currents that contain additive and more complex components. This is depicted by the amplitude difference between BEM and analytical currents on figure 4. Thus, because the shape recognition process estimates the tensor components that makes the model to best fit the measured data (BEM), it necessarily over estimates them. It directly impacts the volume $V$ that is factored out, as shown in (5). To complete this discussion, the shape recognition results including the localization errors of the MUSIC algorithm are shown on figure 11 and in table II. The ellipsoidal objects (numbered 3 to 6 in table I) are faithfully estimated. This shows the ability of the algorithm to estimate the geometrical properties of such objects. On the other hand, the ellipsoids that estimate the cubes are more significantly effected by the localization error. 


\begin{tabular}{|c|c|c|c|c|c|c|}
\hline & $\boldsymbol{x}$ & $\mathbf{x}$ & $\otimes$ & $\bigotimes$ & $\otimes$ & $\bigotimes$ \\
\hline$\hat{x}_{0}^{c}(\mathrm{~cm})$ & 0 & 11.1 & 7 & -4 & 13 & 4.1 \\
\hline$\hat{y}_{0}^{c}(\mathrm{~cm})$ & 5.5 & 7.1 & 6.4 & 8.7 & 4.3 & 6.5 \\
\hline$\hat{\eta}$ & 1.4 & 1.2 & 1.4 & 2.4 & 2.9 & 1.1 \\
\hline$\hat{V}\left(\mathrm{~cm}^{3}\right)$ & 8.6 & 6.9 & 1.1 & 1.2 & 0.49 & 3 \\
\hline$\hat{\theta}_{0}(\mathrm{deg})$ & 81.9 & 5.8 & -12.3 & -74 & 29.2 & - \\
\hline
\end{tabular}

TABLE II: Absolute values of all estimated parameters. For parameters, $\hat{\eta}, \hat{V}$ and $\hat{\theta}_{0}$, values correspond to estimation with localization errors in the shape recognition process. For the sphere, the true location is $\left(x_{0}^{c}, y_{0}^{c}\right)=(4 \mathrm{~cm}, 7 \mathrm{~cm})$.

\section{CONCLUSION}

We have applied to our $U-I$ technology sensor a method proposed in the field of applied mathematics for estimating the location and the geometrical properties of an ellipsoidal object with the electric sense [13]. The simulation results show the applicability of this method in the context of robotics with a very few measurements. Indeed, the principle of localization and geometrical informations separation works. It follows that the ellipsoidal objects' locations and intrinsic properties are well estimated. To a lesser extent, more complex shapes objects can also be estimated by an ellipsoid. Moreover, compare to the algorithm proposed in [10], the method describe in this paper doesn't require any iterative motion loops. Also, it is based on analytical models, instead of numerically identified abacus. Now, these encouraging results have to be confirmed in experimental conditions. In addition, many other alternatives exist for the application of the method and have to be studied. For example, we could use the model of $I_{\text {lat }}$ instead of that of $\delta I_{\mathrm{ax}}$ for the localization with the MUSIC algorithm. Other trajectories that the straight line for the identification phase could also be studied. The estimated positions and geometrical properties of an object with this method could serve as an initial values for a filter in the context of object tracking.

\section{ACKNOWLEDGMENT}

This work was supported by the European Union, by funding the Project: EU H2020 FET-Proactive project "subCULTron", no. 640967. The authors would like to thank Gilles Chabert and Saïd Moussaoui for their precious help on MUSIC algorithm implementation.

\section{REFERENCES}

[1] H. W. Lissmann and K. E. Machin, "The mechanism of object location in Gymnarchus Niloticus and similar fish," Journal of Experimental Biology, vol. 35, no. 2, pp. 451-486, 1958.

[2] C. Assad, B. Rasnow, and P. Stoddard, "Electric organ discharges and electric images during electrolocation," Journal of Experimental Biology, vol. 202, no. 10, pp. 1185-1193, 1999.

[3] J. R. Solberg, K. M. Lynch, and M. A. MacIver, "Active electrolocation for underwater target localization," The International Journal of Robotics Research, vol. 27, no. 5, pp. 529-548, 2008.

[4] F. Boyer, P. Gossiaux, B. Jawad, V. Lebastard, and M. Porez, "Model for a sensor inspired by electric fish," IEEE Transactions on Robotics, vol. 28, no. 2, pp. 492-505, 2012.
[5] N. Servagent, B. Jawad, S. Bouvier, F. Boyer, A. Girin, F. Gomez, V. Lebastard, C. Stefanini, and P.-B. Gossiaux, "Electrolocation sensors in conducting water bio-inspired by electric fish," Sensors Journal, IEEE, vol. 13, no. 5, pp. 1865-1882, 2013.

[6] J. Snyder, Y. Silverman, Y. Bai, and M. MacIver, "Underwater object tracking using electrical impedance tomography," in Intelligent Robots and Systems (IROS), 2012 IEEE/RSJ International Conference on, 2012, pp. 520-525.

[7] Y. Bai, J. Snyder, Y. Silverman, M. Peshkin, and M. MacIver, "Sensing capacitance of underwater objects in bio-inspired electrosense," in 2012 IEEE/RSJ International Conference on Intelligent Robots and Systems (IROS), 2012, pp. 1467-1472.

[8] V. Lebastard, C. Chevallereau, A. Girin, N. Servagent, P.-B. Gossiaux, and F. Boyer, "Environment reconstruction and navigation with electric sense based on a kalman filter," The International Journal of Robotics Research, vol. 32, no. 2, pp. 172-188, 2013.

[9] B. Rasnow, "The effects of simple objects on the electric field of Apteronotus," Journal of Comparative Physiology A, vol. 178, no. 3, pp. 397-411, 1996.

[10] Y. Bai, J. B. Snyder, M. Peshkin, and M. A. MacIver, "Finding and identifying simple objects underwater with active electrosense," The International Journal of Robotics Research, 2015.

[11] T. K. A. Khairuddin and W. R. B. Lionheart, "Fitting ellipsoids to objects by the first order polarization tensor," Malaya Journal of Matematik, vol. 1, pp. 44-53, 2013.

[12] H. Ammari and H. Kang, Polarization and moment tensors, with applications to inverse problems and effective medium theory. SpringerVerlag New York, 2007.

[13] H. Ammari, T. Boulier, and J. Garnier, "Modeling active electrolocation in weakly electric fish," SIAM Journal on Imaging Sciences, vol. 6, no. 1, pp. 285-321, 2013.

[14] R. Schmidt, "Multiple emitter location and signal parameter estimation," IEEE Transactions on Antennas and Propagation, vol. 34, pp. 276-280, 1986.

[15] A. J. Devaney, "Super-resolution Processing of Multi-static Data Using Time Reversal and MUSIC."

[16] M. Porez, V. Lebastard, A. Ijspeert, and F. Boyer, "Multi-physics model of an electric fish-like robot: Numerical aspects and application to obstacle avoidance," in Intelligent Robots and Systems (IROS), 2011 IEEE/RSJ International Conference on, 2011, pp. 1901-1906.

[17] F. Boyer, V. Lebastard, C. Chevallereau, and N. Servagent, "Underwater reflex navigation in confined environment based on electric sense," Robotics, IEEE Transactions on, vol. 29, no. 4, pp. 945-956, 2013.

[18] G. von der Emde, K. Behr, B. Bouton, J. Engelmann, S. Fetz, and C. Folde, "3-dimensional scene perception during active electrolocation in a weakly electric pulse fish," Front Behav Neurosci, vol. 4, 2010.

[19] T. Boulier, "Modélisation de l'électro-localisation active chez les poissons faiblement électriques." Ph.D. dissertation, Ecole Polytechnique, 2013.

[20] H. Krim and M. Viberg, "Two decades of array signal processing research: the parametric approach," Signal Processing Magazine, IEEE, vol. 13, no. 4, pp. 67-94, 1996.

[21] R. A. Horn and C. R. Johnson, Eds., Matrix Analysis. New York, NY, USA: Cambridge University Press, 1986.

[22] L.-P. Song, D. W. Oldenburg, and L. R. Pasion, "Estimating source locations of unexploded ordnance using the multiple signal classification algorithm," GEOPHYSICS, vol. 77, no. 4, pp. WB127-WB135, 2012.

[23] A. J. Devaney, E. A. Marengo, and F. K. Gruber, "Time-reversal-based imaging and inverse scattering of multiply scattering point targets," The Journal of the Acoustical Society of America, vol. 118, no. 5, pp. 3129-3138, 2005.

[24] L. Ljung, System identification (2nd ed.): theory for the user. Prentice Hall PTR, 1999. 